
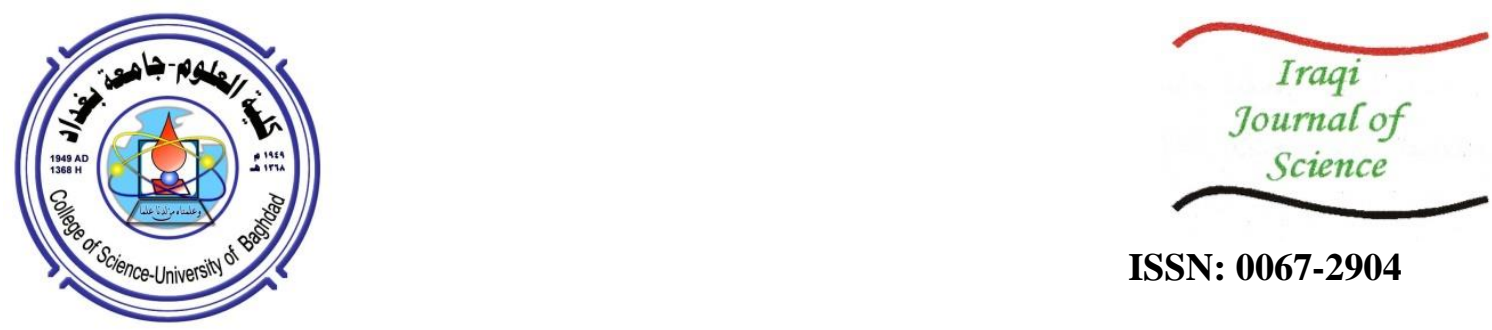

ISSN: 0067-2904

\title{
Application of Two Rowed Weyl Module in the Case of Partition $(7,6)$ and Skew- Partition $(7,6) /(1,0)$
}

\author{
Nejood A. Hatim, Haytham R. Hassan* \\ Department of Mathematics, College of Science, Mustansiriyah University, Baghdad, Iraq
}

Received: 8/9/2020

Accepted: 28/11/2020

\begin{abstract}
The aim of this work is to study the application of Weyl module resolution in the case of two rows, which will be specified in the partition $(7,6)$ and skew- partition $(7,6) /(1,0)$ by using the homological Weyl (i.e. the contracting homotopy and place polarization).
\end{abstract}

Keywords: Divided power algebra, resolution of Weyl module, place polarization, mapping Cone.

$$
\begin{aligned}
& \text { تطبيق مقاس وايل لصفين في حالة التجزئة (7,6) و (7,6)/(1,0)( }
\end{aligned}
$$

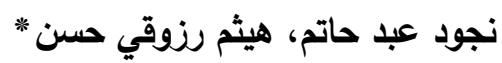

$$
\begin{aligned}
& \text { قسم الرياضيات ، كلية العلوم، جامعه المستصريه، بغداد، العراق }
\end{aligned}
$$

$$
\text { بالتجزئة (6, 7) الغرض من هذا البحث هو دراسة تطبيق تحلل مقاس وايل في حالة الصفين والتئة (1,0)/ }
$$

\section{Introduction}

الدكان).

Let $R$ be a commutative ring with identity , $F$ be a free $R$-module and $D_{b} F$ be the divided power of degree $b$.

Consider the figure below which is associated to the resolution of two-rowed Weyl module $K_{\lambda / \mu} F=\operatorname{Im}\left(d_{\lambda / \mu}^{\prime}\right)$ where $d_{\lambda / \mu}^{\prime}$ is the Weyl map that is described in [1], as follows:

$\lambda / \mu=$

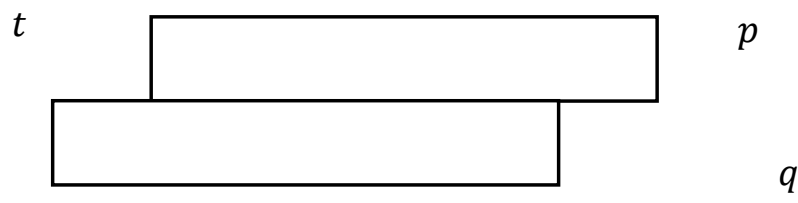

We have:

$$
\sum D_{p+k} \otimes D_{q-k} \stackrel{\square}{\longrightarrow} D_{p} \otimes D_{q} \stackrel{d^{\prime} \lambda / \mu}{\longrightarrow} K_{\lambda / \mu} \rightarrow 0
$$

And by using letter-place algebra, the maps will be explained now as follows: 


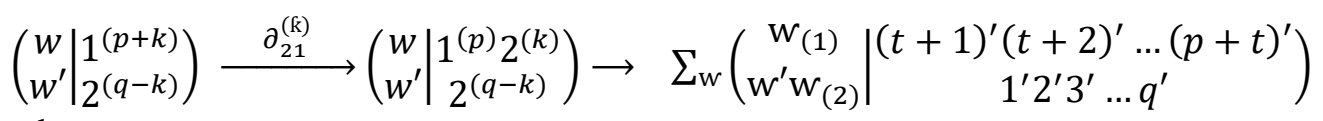

where

and

$$
\mathrm{w} \otimes \mathrm{w}^{\prime} \in D_{p+k} \otimes D_{q-k} \quad, \quad \square=\sum_{k=t+1}^{q} \partial_{21}^{(k)}
$$

$$
d_{\lambda / \mu}^{\prime}=\partial_{q^{\prime} 2} \ldots \partial_{1 / 2} \partial_{(p+\mathrm{t}) / 1} \ldots \partial_{(t+1), 1}
$$

is the composition of place polarization, from positive places $\{1,2\}$ to negative places $\left\{1^{\prime}, 2^{\prime}, \ldots\right.$, $\left.(\mathrm{p}+\mathrm{t})^{\prime}\right\}$.

And, as shown in [2], $\square$ is deliver a component $x \otimes y$ of $D_{p+k} \otimes D_{q-k}$ to $\sum x_{p} \otimes x^{\prime}{ }_{k} y$ where $\sum x_{p} \otimes x^{\prime}{ }_{k}$ is the element of the diagonal of $x$ in $D_{p} \otimes D_{k}$.

Let $Z_{21}$ be the free generator of divided power algebra $D\left(Z_{21}\right)$ in one generator, then the divided power component $Z_{21}^{(k)}$ of degree $\mathrm{K}$ of the free generator $\mathrm{Z}_{21}$ acts on $D_{p+k} \otimes D_{q-k}$ by place polarization of degree $\mathrm{k}$ from place 1 to place 2.

Particularly, the 'graded' algebra 'with identity' $A=D\left(\mathrm{Z}_{21}\right)$ acts on the graded module $M=\sum D_{p+k} \otimes \mathrm{D}_{q-k}=\sum M_{q-k}$, where the degree of the $2^{\text {nd }}$ factor dictates the grading, see $[3,4,5]$.

Therefore, $M$ is a graded left $A$-module, where, for $\mathrm{w}=\mathrm{Z}_{21}^{(k)} \in A$ and $v \in D_{\beta_{1}} \otimes D_{\beta_{2}}$, by definition, we have:

$$
w(v)=\mathrm{Z}_{21}^{(k)}(v)=\partial_{21}^{(k)}(v) .
$$

And if we have $\left(t^{+}\right)$, which is the graded strand of degree $\mathrm{q}$

$$
M_{\bullet}: 0 \rightarrow M_{q-t} \stackrel{\partial_{s}}{\longrightarrow} \ldots \rightarrow M_{l} \stackrel{\partial_{s}}{\longrightarrow} \ldots M_{1} \stackrel{\partial_{s}}{\longrightarrow} M_{0}
$$

of the normalized bar complex, $\operatorname{Bar}(M, A ; \mathrm{S}, \bullet)$, and $\mathrm{S}=\{\mathrm{x}\}$.

By definition, $M_{\bullet}$ is the following complex:

$$
\begin{aligned}
& \sum_{k_{1} \geq 0} \mathrm{Z}_{21}^{\left(t+k_{1}\right)} x \mathrm{Z}_{21}^{\left(k_{2}\right)} x \ldots x \mathrm{Z}_{21}^{\left(k_{l}\right)} x D_{p+t+|\mathrm{k}|} \otimes \mathrm{D}_{q-t-|k|} \stackrel{d_{l}}{\longrightarrow} \\
& \sum_{k_{1} \geq 0} \mathrm{Z}_{21}^{\left(t+k_{1}\right)} x \mathrm{Z}_{21}^{\left(k_{2}\right)} x \ldots x \mathrm{Z}_{21}^{\left(k_{l}-1\right)} \mathcal{H} \mathrm{D}_{P+t+|k|} \otimes D_{q-t-|k|} \stackrel{d_{l-1}}{\longrightarrow} \\
& \ldots \stackrel{d_{1}}{\longrightarrow} \sum_{k_{i} \geq 0} \mathrm{Z}_{21}^{(t+k)} x D_{p+t+|k|} \otimes D_{q-t-k} \stackrel{d_{0}}{\longrightarrow} D_{p} \otimes D_{q}
\end{aligned}
$$

where $|k|=\sum k_{i}$ and $d_{l}$ is the boundary operator $\partial_{\mathcal{H}}$. Notice that (6) illustrates a left complex $\left(\partial_{\mathcal{\varkappa}}^{2}=0\right)$ over the Weyl module in terms of bar complex and letter-place algebra. Furthermore, in (6), when the separator $x$ disappears between a $\mathrm{Z}_{a b}^{(t)}$ and the components in the tensor product of divided powers, this means that $\partial_{a b}^{(t)}$ is applied to the tensor product ( see [1] and [6]).

The authors in [4] and [5] exhibited the terms and the exactness of the Weyl module resolution in the case of partition $(8,7)$ and skew-shape $(8,6) /(2,1)$. In this work, we locate the terms and the exactness of the Weyl module Resolution in the cases of partition $(7,6)$ and skew- partition $(7,6) /(1,0)$.

\section{Application of Weyl Module Resolution in the Case of Partition $(7,6)$.}

\subsection{The Terms of Weyl Module Resolution in the Case of Partition $(7,6)$.}

In this section, we find the term for the resolution of Weyl module in the case of the Partition $(7,6)$.

$$
\begin{aligned}
& M_{0}=\mathrm{D}_{7} \otimes \mathrm{D}_{6} \\
& M_{1}=\mathrm{Z}_{21} \mathcal{H} \mathrm{D}_{8} \otimes \mathrm{D}_{5} \quad \oplus \mathrm{Z}_{21}^{(2)} \mathcal{H} \mathrm{D}_{9} \otimes \mathrm{D}_{4} \oplus \mathrm{Z}_{21}^{(3)} \mathcal{H} \mathrm{D}_{10} \otimes \mathrm{D}_{3} \oplus \mathrm{Z}_{21}^{(4)} \mathcal{H} \mathrm{D}_{11} \otimes \mathrm{D}_{2}
\end{aligned}
$$

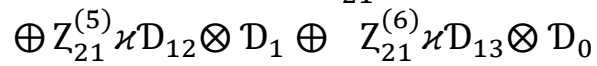

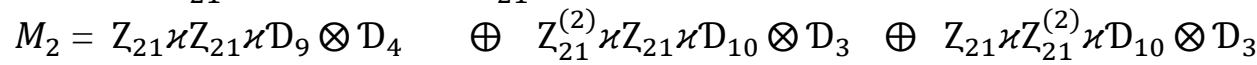

$$
\begin{aligned}
& \oplus \mathrm{Z}_{21}^{(3)} \varkappa \mathrm{Z}_{21} \varkappa \mathrm{D}_{11} \otimes \mathrm{D}_{2} \oplus \mathrm{Z}_{21} \varkappa \mathrm{Z}_{21}^{(3)} \varkappa \mathrm{D}_{11} \otimes \mathrm{D}_{2} \oplus \mathrm{Z}_{21}^{(2)} \varkappa \mathrm{Z}_{21}^{(2)} \varkappa \mathrm{D}_{11} \otimes \mathrm{D}_{2}
\end{aligned}
$$

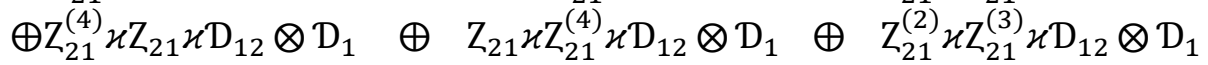




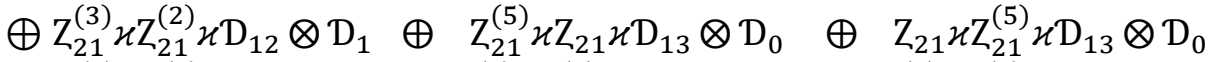

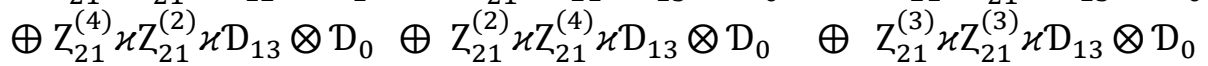
$M_{3}=\mathrm{Z}_{21} \varkappa \mathrm{Z}_{21} \varkappa \mathrm{Z}_{21} \varkappa \mathrm{D}_{10} \otimes \mathrm{D}_{3} \oplus \mathrm{Z}_{21}^{(2)} \varkappa \mathrm{Z}_{21} \varkappa \mathrm{Z}_{21} \varkappa \mathrm{D}_{11} \otimes \mathrm{D}_{2} \oplus \mathrm{Z}_{21} \varkappa \mathrm{Z}_{21}^{(2)} \varkappa \mathrm{Z}_{21} \varkappa \mathrm{D}_{11} \otimes \mathrm{D}_{2}$

$\oplus \mathrm{Z}_{21} \varkappa \mathrm{Z}_{21} \varkappa \mathrm{Z}_{21}^{(2)} \varkappa \mathrm{D}_{11} \otimes \mathrm{D}_{2} \oplus \mathrm{Z}_{21}^{(3)} \varkappa \mathrm{Z}_{21} \varkappa \mathrm{Z}_{21} \varkappa \mathrm{D}_{12} \otimes \mathrm{D}_{1} \oplus \mathrm{Z}_{21} \varkappa \mathrm{Z}_{21}^{(3)} \varkappa \mathrm{Z}_{21} \varkappa \mathrm{D}_{12} \otimes \mathrm{D}_{1}$

$\oplus \mathrm{Z}_{21} \varkappa \mathrm{Z}_{21} \varkappa \mathrm{Z}_{21}^{(3)} \varkappa \mathrm{D}_{12} \otimes \mathrm{D}_{1} \oplus \mathrm{Z}_{21}^{(2)} \varkappa \mathrm{Z}_{21}^{(2)} \varkappa \mathrm{Z}_{21} \varkappa \mathrm{D}_{12} \otimes \mathrm{D}_{1} \oplus \mathrm{Z}_{21} \varkappa \mathrm{Z}_{21}^{(2)} \varkappa \mathrm{Z}_{21}^{(2)} \varkappa \mathrm{D}_{12} \otimes \mathrm{D}_{1}$

$\oplus \mathrm{Z}_{21}^{(2)} \varkappa \mathrm{Z}_{21} \varkappa \mathrm{Z}_{21}^{(2)} \varkappa \mathrm{D}_{12} \otimes \mathrm{D}_{1} \oplus \mathrm{Z}_{21}^{(4)} \varkappa \mathrm{Z}_{21} \varkappa \mathrm{Z}_{21} \varkappa \mathrm{D}_{13} \otimes \mathrm{D}_{0} \oplus \mathrm{Z}_{21} \varkappa \mathrm{Z}_{21}^{(4)} \varkappa \mathrm{Z}_{21} \varkappa \mathrm{D}_{13} \otimes \mathrm{D}_{0}$

$\oplus \mathrm{Z}_{21} \varkappa \mathrm{Z}_{21} \varkappa \mathrm{Z}_{21}^{(4)} \varkappa \mathrm{D}_{13} \otimes \mathrm{D}_{0} \oplus \mathrm{Z}_{21}^{(3)} \varkappa \mathrm{Z}_{21}^{(2)} \varkappa \mathrm{Z}_{21} \varkappa \mathrm{D}_{13} \otimes \mathrm{D}_{0} \oplus \mathrm{Z}_{21}^{(3)} \varkappa \mathrm{Z}_{21} \varkappa \mathrm{Z}_{21}^{(2)} \varkappa \mathrm{D}_{13} \otimes \mathrm{D}_{0}$

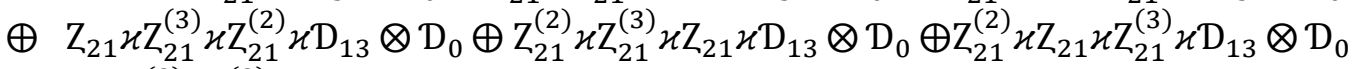

$\oplus \mathrm{Z}_{21} \varkappa \mathrm{Z}_{21}^{(2)} \varkappa \mathrm{Z}_{21}^{(3)} \varkappa \mathrm{D}_{13} \otimes \mathrm{D}_{0}$

$M_{4}=\mathrm{Z}_{21} \varkappa \mathrm{Z}_{21} \varkappa \mathrm{Z}_{21} \varkappa \mathrm{Z}_{21} \varkappa \mathrm{D}_{11} \otimes \mathrm{D}_{2} \oplus \mathrm{Z}_{21}^{(2)} \varkappa \mathrm{Z}_{21} \varkappa \mathrm{Z}_{21} \varkappa \mathrm{Z}_{21} \varkappa \mathrm{D}_{12} \otimes \mathrm{D}_{1}$

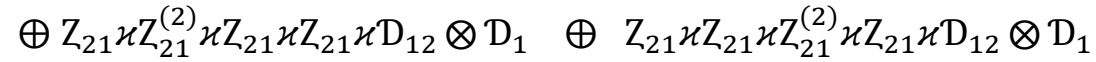

$\oplus \mathrm{Z}_{21} \varkappa \mathrm{Z}_{21} \varkappa \mathrm{Z}_{21} \varkappa \mathrm{Z}_{21}^{(2)} \varkappa \mathrm{D}_{12} \otimes \mathrm{D}_{1} \oplus \mathrm{Z}_{21}^{(2)} \varkappa \mathrm{Z}_{21}^{(2)} \varkappa \mathrm{Z}_{21} \varkappa \mathrm{Z}_{21} \varkappa \mathrm{D}_{13} \otimes \mathrm{D}_{0}$

$\oplus \mathrm{Z}_{21}^{(2)} \varkappa \mathrm{Z}_{21} \varkappa \mathrm{Z}_{21}^{(2)} \varkappa \mathrm{Z}_{21} \varkappa \mathrm{D}_{13} \otimes \mathrm{D}_{0} \oplus \mathrm{Z}_{21}^{(2)} \varkappa \mathrm{Z}_{21} \varkappa \mathrm{Z}_{21} \varkappa \mathrm{Z}_{21}^{(2)} \varkappa \mathrm{D}_{13} \otimes \mathrm{D}_{0}$

$\oplus \mathrm{Z}_{21} \varkappa \mathrm{Z}_{21}^{(2)} \varkappa \mathrm{Z}_{21}^{(2)} \varkappa \mathrm{Z}_{21} \varkappa \mathrm{D}_{13} \otimes \mathrm{D}_{0} \oplus \mathrm{Z}_{21} \varkappa \mathrm{Z}_{21}^{(2)} \varkappa \mathrm{Z}_{21} \varkappa \mathrm{Z}_{21}^{(2)} \varkappa \mathrm{D}_{13} \otimes \mathrm{D}_{0}$

$\oplus \mathrm{Z}_{21} \varkappa \mathrm{Z}_{21} \varkappa \mathrm{Z}_{21}^{(2)} \varkappa \mathrm{Z}_{21}^{(2)} \varkappa \mathrm{D}_{13} \otimes \mathrm{D}_{0} \oplus \mathrm{Z}_{21}^{(3)} \varkappa \mathrm{Z}_{21} \varkappa \mathrm{Z}_{21} \varkappa \mathrm{Z}_{21} \varkappa \mathrm{D}_{13} \otimes \mathrm{D}_{0}$

$\oplus \mathrm{Z}_{21} \varkappa \mathrm{Z}_{21}^{(3)} \varkappa \mathrm{Z}_{21} \varkappa \mathrm{Z}_{21} \varkappa \mathrm{D}_{13} \otimes \mathrm{D}_{0} \oplus \mathrm{Z}_{21} \varkappa \mathrm{Z}_{21} \varkappa \mathrm{Z}_{21}^{(3)} \varkappa \mathrm{Z}_{21} x \mathrm{D}_{13} \otimes \mathrm{D}_{0}$

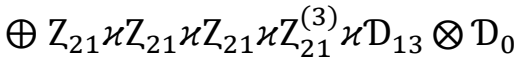

$M_{5}=\mathrm{Z}_{21} \varkappa \mathrm{Z}_{21} \varkappa \mathrm{Z}_{21} \varkappa \mathrm{Z}_{21} \varkappa \mathrm{Z}_{21} \varkappa \mathrm{D}_{12} \otimes \mathrm{D}_{1} \oplus \mathrm{Z}_{21}^{(2)} \varkappa \mathrm{Z}_{21} \varkappa \mathrm{Z}_{21} \varkappa \mathrm{Z}_{21} \varkappa \mathrm{Z}_{21} \varkappa \mathrm{D}_{13} \otimes \mathrm{D}_{0}$

$\oplus \mathrm{Z}_{21} \varkappa \mathrm{Z}_{21}^{(2)} \varkappa \mathrm{Z}_{21} \varkappa \mathrm{Z}_{21} \varkappa \mathrm{Z}_{21} \varkappa \mathrm{D}_{13} \otimes \mathrm{D}_{0} \oplus \mathrm{Z}_{21} \varkappa \mathrm{Z}_{21} \varkappa \mathrm{Z}_{21}^{(2)} \varkappa \mathrm{Z}_{21} \varkappa \mathrm{Z}_{21} \varkappa \mathrm{D}_{13} \otimes \mathrm{D}_{0}$

$\oplus \mathrm{Z}_{21} \varkappa \mathrm{Z}_{21} \varkappa \mathrm{Z}_{21}^{(2)} \varkappa \mathrm{Z}_{21} \varkappa \mathrm{Z}_{21} \varkappa \mathrm{D}_{13} \otimes \mathrm{D}_{0} \oplus \mathrm{Z}_{21} \varkappa \mathrm{Z}_{21} \varkappa \mathrm{Z}_{21} \varkappa \mathrm{Z}_{21}^{(2)} \varkappa \mathrm{Z}_{21} \varkappa \mathrm{D}_{13} \otimes \mathrm{D}_{0}$

$\oplus \mathrm{Z}_{21} \varkappa \mathrm{Z}_{21} \varkappa \mathrm{Z}_{21} \varkappa \mathrm{Z}_{21} \varkappa \mathrm{Z}_{21}^{(2)} \varkappa \mathrm{D}_{13} \otimes \mathrm{D}_{0}$

$M_{6}=\mathrm{Z}_{21} \varkappa \mathrm{Z}_{21} \varkappa \mathrm{Z}_{21} \varkappa \mathrm{Z}_{21} \varkappa \mathrm{Z}_{21} \varkappa \mathrm{Z}_{21} \varkappa \mathrm{D}_{13} \otimes \mathrm{D}_{0}$

\subsection{The Exactness of Weyl Resolution in the Case of Partition $(7,6)$}

This section explains the building of contracting homotopies $\left\{S_{i}\right\}$, where $\mathrm{i}=1,2, \ldots, 5$.

We define the $S_{i}$ map as follows:

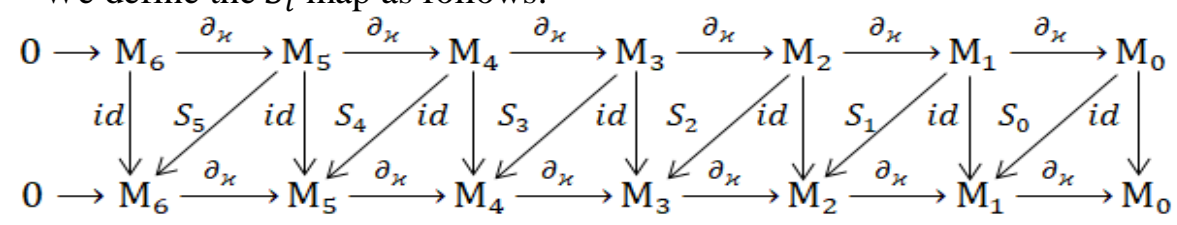

$S_{0}: M_{0} \rightarrow M_{1}$

$S_{0}\left(\left(\begin{array}{c|c}w & 1^{(7)} 2^{(k)} \\ w^{\prime} & 2^{(6-k)}\end{array}\right)\right)=\left\{\begin{array}{c}\mathrm{Z}_{21}^{(k)} \mathcal{\varkappa}\left(\begin{array}{c|c}w & 1^{(7+\mathrm{k})} \\ w^{\prime} & 2^{(6-\mathrm{k})}\end{array}\right) \quad \text {;if } k>0 \\ 0 \quad \text { if } k \leq 0\end{array}\right.$

$S_{1}: M_{1} \rightarrow M_{2}$

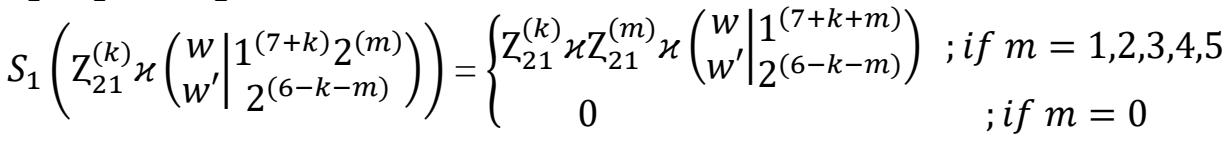

$S_{2}: M_{2} \rightarrow M_{3}$

$S_{2}\left(\mathrm{Z}_{21}^{\left(k_{1}\right)} \mathcal{H} \mathrm{Z}_{21}^{\left(k_{2}\right)} \mathcal{X}\left(\begin{array}{c|c}w & 1^{(7+|m|)} 2^{(m)} \\ w^{\prime} & 2^{(6-|k|-m)}\end{array}\right)\right)=$

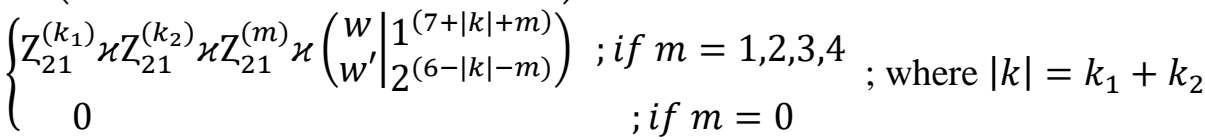

$S_{3}: M_{3} \rightarrow M_{4}$

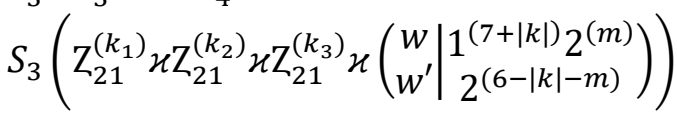




$$
=\left\{\begin{array}{cc}
\mathrm{Z}_{21}^{\left(k_{1}\right)} \mathcal{H} \mathrm{Z}_{21}^{\left(k_{2}\right)} \mathcal{H} \mathrm{Z}_{21}^{\left(k_{3}\right)} \mathcal{H} \mathrm{Z}_{21}^{(m)} \mathcal{H}\left(\left.\begin{array}{c}
w \\
w^{\prime}
\end{array}\right|_{2^{(6-|k|-m)}} ^{(7+|k|+m)}\right) ; \text { if } m=1,2,3 \\
0 \quad \text {; if } m=0
\end{array} ;\right.
$$

where $|k|=k_{1}+k_{2}+k_{3}$

$S_{4}: M_{4} \rightarrow M_{5}$

$S_{4}\left(\mathrm{Z}_{21}^{\left(k_{1}\right)} \mathcal{H} \mathrm{Z}_{21}^{\left(k_{2}\right)} \mathcal{H} \mathrm{Z}_{21}^{\left(k_{3}\right)} \mathcal{H} \mathrm{Z}_{21}^{\left(k_{4}\right)} \mathcal{\varkappa}\left(\begin{array}{c|c}w & 1^{(7+|k|)} 2^{(m)} \\ w^{\prime} & 2^{(6-|k|-m)}\end{array}\right)\right)$

$$
= \begin{cases}\mathrm{Z}_{21}^{\left(k_{1}\right)} \mathcal{H} \mathrm{Z}_{21}^{\left(k_{2}\right)} \mathcal{H} \mathrm{Z}_{21}^{\left(k_{3}\right)} \mathcal{H} \mathrm{Z}_{21}^{\left(k_{4}\right)} \mathcal{H} \mathrm{Z}_{21}^{(m)} \mathcal{\varkappa}\left(\begin{array}{c}
w \\
w^{\prime} \mid 1_{2^{(6-|k|-m)}}^{(7+|k|+m)}
\end{array}\right) & \text {; if } m=1,2 \\
0 & \text {; if } m=0\end{cases}
$$

where $|k|=k_{1}+k_{2}+k_{3}+k_{4}$

$S_{5}: M_{5} \rightarrow M_{6}$

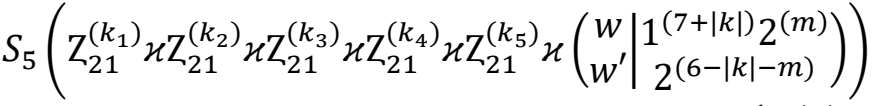

$$
\begin{aligned}
& = \begin{cases}\mathrm{Z}_{21}^{\left(k_{1}\right)} \mathcal{H} \mathrm{Z}_{21}^{\left(k_{2}\right)} \mathcal{H} \mathrm{Z}_{21}^{\left(k_{3}\right)} \mathcal{H} \mathrm{Z}_{21}^{(k)} \mathcal{H} \mathrm{Z}_{21}^{\left(k_{5}\right)} \mathcal{H} \mathrm{Z}_{21}^{(m)} \mathcal{H}\left(\begin{array}{c}
w \\
\left.w^{\prime}\right|_{2^{(6-|k|-m)}} ^{(7+|k|+m)}
\end{array}\right) & \text {; if } m=1 \\
0 & \text {;if } m=0\end{cases}
\end{aligned}
$$

where $|k|=k_{1}+k_{2}+k_{3}+k_{4}+k_{5}$

$S_{0} \partial_{\mathcal{H}}\left(\mathrm{Z}_{21}^{(k)} \mathcal{\varkappa}\left(\begin{array}{c|c}w & 1^{(7+k)} 2^{(m)} \\ w^{\prime} & 2^{(6-k-m)}\end{array}\right)\right)=S_{0} \partial_{21}^{(k)}\left(\begin{array}{c|c}w & 1^{(7+k)} 2^{(m)} \\ w^{\prime} & 2^{(6-k-m)}\end{array}\right)$

$=\left(\begin{array}{c}k+m \\ m\end{array}\right) \mathrm{Z}_{21}^{(k+m)} \mathcal{H}\left(\begin{array}{c}w \\ w^{\prime}\end{array} 1^{(7+k+m)}\left(\begin{array}{l}(6-k-m)\end{array}\right)\right.$

and

$$
\begin{aligned}
& \partial_{\mathcal{H}} S_{1}\left(\mathrm{Z}_{21}^{(k)} \mathcal{\varkappa}\left(\begin{array}{c|c}
w & 1^{(7+k)} 2^{(m)} \\
w^{\prime} & 2^{(6-k-m)}
\end{array}\right)\right)=\partial_{\mathcal{\varkappa}}\left(\mathrm{Z}_{21}^{(k)} \mathcal{H} \mathrm{Z}_{21}^{(m)} \mathcal{\varkappa}\left(\begin{array}{c|c}
w & 1^{(7+k+m)} \\
w^{\prime} & 2^{(6-k-m)}
\end{array}\right)\right)
\end{aligned}
$$

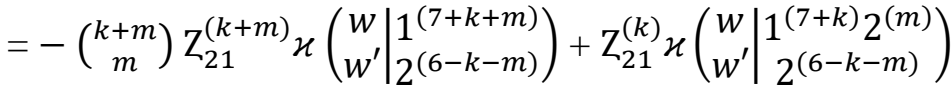

$$
\begin{aligned}
& =\mathrm{Z}_{21}^{(k)} \mathcal{H}\left(\begin{array}{c|c}
w & 1^{(7+k)} 2^{(m)} \\
w^{\prime} & 2^{(6-k-m)}
\end{array}\right)
\end{aligned}
$$

It is clear that $\quad S_{0} \partial_{\mathcal{\varkappa}}+\partial_{\mathcal{\varkappa}} S_{1}=i d_{M_{1}}$.

$S_{1} \partial_{\mathcal{X}}\left(\mathrm{Z}_{21}^{\left(k_{1}\right)} \mathcal{H} \mathrm{Z}_{21}^{\left(k_{2}\right)} \mathcal{X}\left(\begin{array}{c|c}w & 1^{(7+|k|)} 2^{(m)} \\ w^{\prime} & 2^{(6-|k|-m)}\end{array}\right)\right)$

$=S_{1}\left(-\left(\begin{array}{c}|k| \\ k_{2}\end{array}\right) \mathrm{Z}_{21}^{|k|} \mathcal{\varkappa}\left(\begin{array}{c|c}w & 1^{(7+|k|)} 2^{(m)} \\ w^{\prime} & 2^{(6-|k|-m)}\end{array}\right)+\mathrm{Z}_{21}^{\left(k_{1}\right)} \mathcal{H} \partial_{21}^{\left(k_{2}\right)}\left(\begin{array}{c|c}w & 1^{(7+|k|)} 2^{(m)} \\ w^{\prime} & 2^{(6-|k|-m)}\end{array}\right)\right)$

$=-\left(\begin{array}{c}|k| \\ k_{2}\end{array}\right) \mathrm{Z}_{21}^{|k|} \mathcal{H} \mathrm{Z}_{21}^{(m)} \mathcal{X}\left(\begin{array}{c|c}w & 1^{(7+|k|+m)} \\ w^{\prime} & 2^{(6-|k|-m)}\end{array}\right)+$

$\left(\begin{array}{c}k_{2}+m \\ m\end{array}\right) \mathrm{Z}_{21}^{\left(k_{1}\right)} \mathcal{H} \mathrm{Z}_{21}^{\left(k_{2}+m\right)} \mathcal{H}\left(\begin{array}{c}w \\ w^{\prime} \\ 2^{(6-|k|-m)}\end{array}\right)$

and

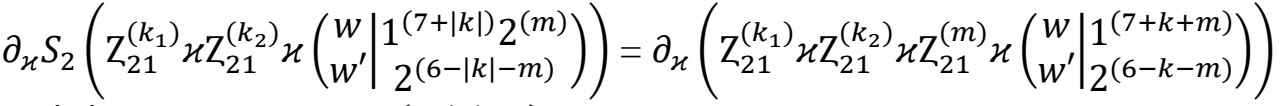

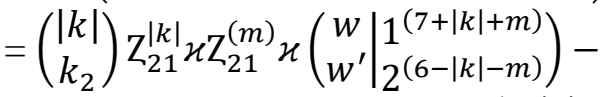

$$
\begin{aligned}
& \left(\begin{array}{c}
k_{2}+m \\
m
\end{array}\right) \mathrm{Z}_{21}^{\left(k_{1}\right)} \mathcal{H} \mathrm{Z}_{21}^{\left(k_{2}+m\right)} \mathcal{H}\left(\begin{array}{c|c}
w & 1^{(7+|k|+m)} \\
w^{\prime} & 2^{(6-|k|-m)}
\end{array}\right)+\mathrm{Z}_{21}^{\left(k_{1}\right)} \mathcal{H} \mathrm{Z}_{21}^{\left(k_{2}\right)}\left(\begin{array}{c|c}
\mathrm{W} & 1^{(7+|k|)} 2^{(m)} \\
\mathrm{w}^{\prime} & 2^{(6-|k|-m)}
\end{array}\right),
\end{aligned}
$$

where $|k|=k_{1}+k_{2}$.

It is clear that $\quad S_{1} \partial_{\mathcal{\varkappa}}+\partial_{\mathcal{\varkappa}} S_{2}=i d_{M_{2}}$.

$S_{2} \partial_{\mathcal{H}}\left(\mathrm{Z}_{21}^{\left(k_{1}\right)} \mathcal{H} \mathrm{Z}_{21}^{\left(k_{2}\right)} \mathcal{H} \mathrm{Z}_{21}^{\left(k_{3}\right)} \mathcal{\varkappa}\left(\begin{array}{c|c}w & 1^{(7+|k|)} 2^{(m)} \\ w^{\prime} & 2^{(6-|k|-m)}\end{array}\right)\right)$ 


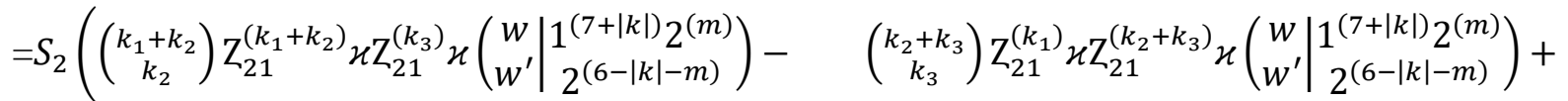

$$
\begin{aligned}
& \left.\mathrm{Z}_{21}^{\left(k_{1}\right)} \mathcal{H} \mathrm{Z}_{21}^{\left(k_{2}\right)} \mathcal{H} \mathrm{Z}_{21}^{\left(k_{3}\right)}\left(\begin{array}{c|c}
w & 1^{(7+|k|)} 2^{(m)} \\
w^{\prime} & 2^{(6-|k|-m)}
\end{array}\right)\right) \\
& =\left(\begin{array}{c}
k_{1}+k_{2} \\
k_{2}
\end{array}\right) \mathrm{Z}_{21}^{\left(k_{1}+k_{2}\right)} \mathcal{H} \mathrm{Z}_{21}^{\left(k_{3}\right)} \mathcal{H} \mathrm{Z}_{21}^{(m)} \mathcal{\varkappa}\left(\begin{array}{c|c}
w & 1^{(7+|k|+m)} \\
w^{\prime} & 2^{(6-|k|-m)}
\end{array}\right)- \\
& \left(\begin{array}{c}
k_{2}+k_{3} \\
k_{3}
\end{array}\right) \mathrm{Z}_{21}^{\left(k_{1}\right)} \mathcal{H} \mathrm{Z}_{21}^{\left(k_{2}+k_{3}\right)} \mathcal{H} \mathrm{Z}_{21}^{(m)} \mathcal{H}\left(\begin{array}{c|c}
w & 1^{(7+|k|+m)} \\
w^{\prime} & 2^{(6-|k|-m)}
\end{array}\right)+ \\
& \left(\begin{array}{c}
k_{3}+m \\
m
\end{array}\right) \mathrm{Z}_{21}^{\left(k_{1}\right)} \mathcal{H} \mathrm{Z}_{21}^{\left(k_{2}\right)} \mathcal{H} \mathrm{Z}_{21}^{\left(k_{3}+m\right)} \mathcal{\varkappa}\left(\begin{array}{c|c}
w & 1^{(7+|k|+m)} \\
w^{\prime} & 2^{(6-|k|-m)}
\end{array}\right),
\end{aligned}
$$

and

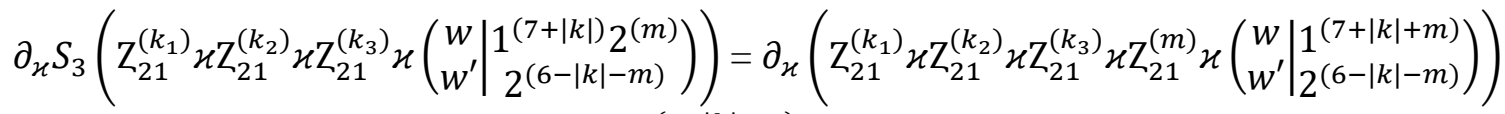

$$
\begin{aligned}
& =-\left(\begin{array}{c}
k_{1}+k_{2} \\
k_{2}
\end{array}\right) \mathrm{Z}_{21}^{\left(k_{1}+k_{2}\right)} \mathcal{H} \mathrm{Z}_{21}^{\left(k_{3}\right)} \mathcal{H} \mathrm{Z}_{21}^{(m)} \mathcal{\varkappa}\left(\begin{array}{c|c|c|}
w & 1^{(7+|k|+m)} \\
w^{\prime} & 2^{(6-|k|-m)}
\end{array}\right)+
\end{aligned}
$$

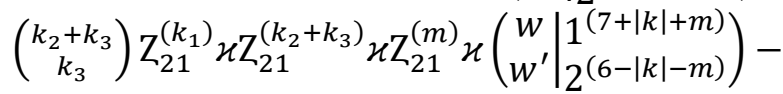

$$
\begin{aligned}
& \left(\begin{array}{c}
k_{3}+m \\
m
\end{array}\right) \mathrm{Z}_{21}^{\left(k_{1}\right)} \mathcal{H} \mathrm{Z}_{21}^{\left(k_{2}\right)} \mathcal{H} \mathrm{Z}_{21}^{\left(k_{3}+m\right)} x\left(\begin{array}{c}
w \\
w^{\prime} \\
2^{(6-|k|-m)}
\end{array}\right)+ \\
& \mathrm{Z}_{21}^{\left(k_{1}\right)} \mathcal{H} \mathrm{Z}_{21}^{\left(k_{2}\right)} \mathcal{H} \mathrm{Z}_{21}^{\left(k_{3}\right)} \mathcal{H} \partial_{21}^{(m)}\left(\begin{array}{c|c}
w & 1^{(7+|k|+m)} \\
w^{\prime} & 2^{(6-|k|-m)}
\end{array}\right) \\
& =-\left(\begin{array}{c}
k_{1}+k_{2} \\
k_{2}
\end{array}\right) \mathrm{Z}_{21}^{\left(k_{1}+k_{2}\right)} \mathcal{H} \mathrm{Z}_{21}^{\left(k_{3}\right)} \mathcal{H} \mathrm{Z}_{21}^{(m)} \mathcal{X}\left(\begin{array}{c}
w \\
w^{\prime}
\end{array}{\begin{array}{l}
1^{(7+|k|+m)} \\
(6-|k|-m)
\end{array}}^{(7+\mid+m)}\right)+
\end{aligned}
$$

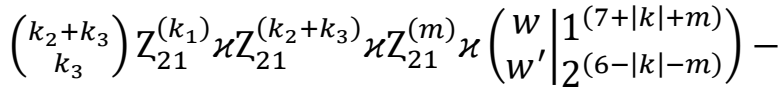

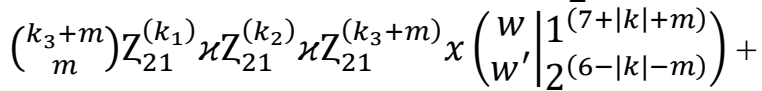

$$
\begin{aligned}
& \mathrm{Z}_{21}^{\left(k_{1}\right)} \mathcal{H} \mathrm{Z}_{21}^{\left(k_{2}\right)} \mathcal{H} \mathrm{Z}_{21}^{\left(k_{3}\right)} \mathcal{X}\left(\begin{array}{c|c}
w & 1^{(7+|k|)} 2^{(m)} \\
w^{\prime} & 2^{(6-|k|-m)}
\end{array}\right),
\end{aligned}
$$

where $|k|=k_{1}+k_{2}+k_{3}$.

It is clear that $\quad S_{2} \partial_{\mathcal{\varkappa}}+\partial_{\mathcal{\varkappa}} S_{3}=i d_{M_{3}}$.

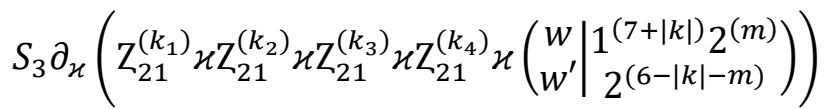

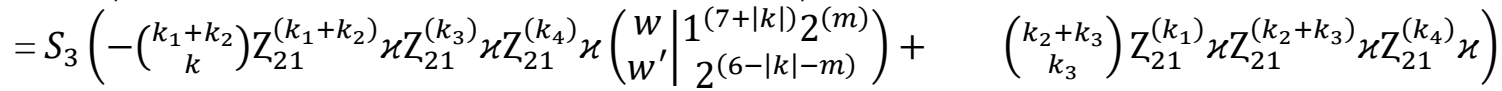

$$
\begin{aligned}
& \left(\begin{array}{c|c}
w & 1^{(7+|k|)} 2^{(m)} \\
w^{\prime} & 2^{(6-|k|-m)}
\end{array}\right)\left(\begin{array}{c}
k_{3}+k_{4} \\
k_{4}
\end{array}\right) \mathrm{Z}_{21}^{\left(k_{1}\right)} \mathcal{H} \mathrm{Z}_{21}^{\left(k_{2}\right)} \mathcal{H} \mathrm{Z}_{21}^{\left(k_{3}+k_{4}\right)} \mathcal{H}\left(\begin{array}{c|c}
w & 1^{(7+|k|)} 2^{(m)} \\
w^{\prime} & 2^{(6-|k|-m)}
\end{array}\right)+
\end{aligned}
$$

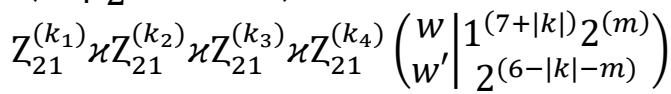

$$
\begin{aligned}
& =-\left(\begin{array}{c}
k_{1}+k_{2} \\
k_{2}
\end{array}\right) \mathrm{Z}_{21}^{\left(k_{1}+k_{2}\right)} \mathcal{H} \mathrm{Z}_{21}^{\left(k_{3}\right)} \mathcal{H} \mathrm{Z}_{21}^{\left(k_{4}\right)} \mathcal{H} \mathrm{Z}_{21}^{(m)} \mathcal{\varkappa}\left(\begin{array}{c|c|c}
w & 1^{(7+|k|+m)} \\
w^{\prime} & 2^{(6-|k|-m)}
\end{array}\right)+ \\
& \left(\begin{array}{c}
k_{2}+k_{3} \\
k_{3}
\end{array}\right) \mathrm{Z}_{21}^{\left(k_{1}\right)} \mathcal{H} \mathrm{Z}_{21}^{\left(k_{2}+k_{3}\right)} \mathcal{H} \mathrm{Z}_{21}^{\left(k_{4}\right)} \mathcal{H} \mathrm{Z}_{21}^{(m)} \mathcal{\varkappa}\left(\begin{array}{c|c}
w & 1^{(7+|k|+m)} \\
w^{\prime} & 2^{(6-|k|-m)}
\end{array}\right)-
\end{aligned}
$$

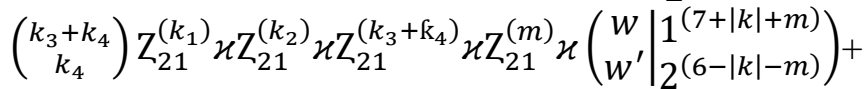

$$
\begin{aligned}
& \left(\begin{array}{c}
k_{4}+m \\
m
\end{array}\right) \mathrm{Z}_{21}^{\left(k_{1}\right)} \mathcal{H} \mathrm{Z}_{21}^{\left(k_{2}\right)} \mathcal{H} \mathrm{Z}_{21}^{\left(k_{3}\right)} \mathcal{H} \mathrm{Z}_{21}^{\left(k_{4}+m\right)} \mathcal{H}\left(\begin{array}{c}
w \\
w^{\prime} \\
2^{(6-|k|-m)}
\end{array}\right),
\end{aligned}
$$

and

$$
\begin{aligned}
& \partial_{\mathcal{H}} S_{4}\left(\mathrm{Z}_{21}^{\left(k_{1}\right)} \mathcal{H} \mathrm{Z}_{21}^{\left(k_{2}\right)} \mathcal{H} \mathrm{Z}_{21}^{\left(k_{3}\right)} \mathcal{H} \mathrm{Z}_{21}^{\left(k_{4}\right)} x\left(\begin{array}{c|c}
w & 1^{(7+|k|)} 2^{(m)} \\
w^{\prime} & 2^{(6-|k|-m)}
\end{array}\right)\right) \\
& =\partial_{\mathcal{H}}\left(\mathrm{Z}_{21}^{\left(k_{1}\right)} \mathcal{H} \mathrm{Z}_{21}^{\left(k_{2}\right)} \mathcal{H} \mathrm{Z}_{21}^{\left(k_{3}\right)} \mathcal{H} \mathrm{Z}_{21}^{\left(k_{4}\right)} \mathcal{H} \mathrm{Z}_{21}^{(m)} \mathcal{\varkappa}\left(\begin{array}{c|c}
w & 1^{(7+|k|+m)} \\
w^{\prime} & 2^{(6-|k|-m)}
\end{array}\right)\right)
\end{aligned}
$$




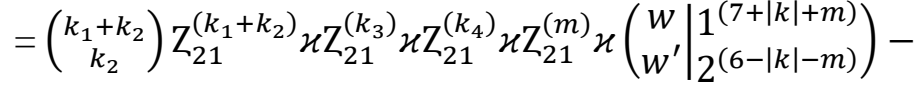

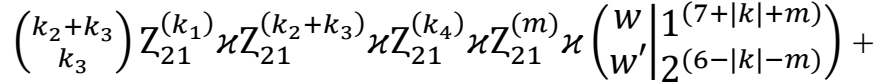

$$
\begin{aligned}
& \left(\begin{array}{c}
k_{3}+k_{4} \\
k_{4}
\end{array}\right) \mathrm{Z}_{21}^{\left(k_{1}\right)} \varkappa \mathrm{Z}_{21}^{\left(k_{2}\right)} \mathcal{H} \mathrm{Z}_{21}^{\left(k_{3}+k_{4}\right)} \varkappa \mathrm{Z}_{21}^{(m)} \varkappa\left(\begin{array}{c}
w \\
w^{\prime} \mid 1_{2^{(6-|k|-m)}}^{(7+|k|+m)}
\end{array}\right)- \\
& \left(\begin{array}{c}
k_{4}+m \\
m
\end{array}\right) \mathrm{Z}_{21}^{\left(k_{1}\right)} \mathcal{H} \mathrm{Z}_{21}^{\left(k_{2}\right)} \mathcal{H} \mathrm{Z}_{21}^{\left(k_{3}\right)} \mathcal{H} \mathrm{Z}_{21}^{\left(k_{4}+m\right)} \mathcal{\varkappa}\left(\begin{array}{c|c}
w & 1^{(7+|k|+m)} \\
w^{\prime} & 2^{(6-|k|-m)}
\end{array}\right)+ \\
& \mathrm{Z}_{21}^{\left(k_{1}\right)} \varkappa \mathrm{Z}_{21}^{\left(k_{2}\right)} \varkappa \mathrm{Z}_{21}^{\left(k_{3}\right)} \varkappa \mathrm{Z}_{21}^{\left(k_{4}\right)} \varkappa \partial_{21}^{(m)}\left(\begin{array}{c|c}
w & 1^{(7+|k|+m)} \\
w^{\prime} & 2^{(6-|k|-m)}
\end{array}\right)
\end{aligned}
$$

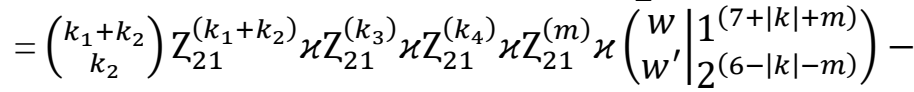

$$
\begin{aligned}
& \left(\begin{array}{c}
k_{2}+k_{3} \\
k_{3}
\end{array}\right) \mathrm{Z}_{21}^{\left(k_{1}\right)} \mathcal{H} \mathrm{Z}_{21}^{\left(k_{2}+k_{3}\right)} \mathcal{H} \mathrm{Z}_{21}^{\left(k_{4}\right)} \mathcal{H} \mathrm{Z}_{21}^{(m)} \mathcal{\varkappa}\left(\begin{array}{c|c}
w & 1^{(7+|k|+m)} \\
w^{\prime} & 2^{(6-|k|-m)}
\end{array}\right)+ \\
& \left(\begin{array}{c}
k_{3}+k_{4} \\
k_{4}
\end{array}\right) \mathrm{Z}_{21}^{\left(k_{1}\right)} \mathcal{H} \mathrm{Z}_{21}^{\left(k_{2}\right)} \mathcal{H} \mathrm{Z}_{21}^{\left(k_{3}+k_{4}\right)} \mathcal{H} \mathrm{Z}_{21}^{(m)} \mathcal{\varkappa}\left(\begin{array}{c|c}
w & 1^{(7+|k|+m)} \\
w^{\prime} & 2^{(6-|k|-m)}
\end{array}\right)- \\
& \left(\begin{array}{c}
k_{4}+m \\
m
\end{array}\right) \mathrm{Z}_{21}^{\left(k_{1}\right)} \mathcal{H} \mathrm{Z}_{21}^{\left(k_{2}\right)} \mathcal{H} \mathrm{Z}_{21}^{\left(k_{3}\right)} \mathcal{H} \mathrm{Z}_{21}^{\left(k_{4}+m\right)} \mathcal{X}\left(\begin{array}{c|c}
w & 1^{(7+|k|+m)} \\
w^{\prime} & 2^{(6-|k|-m)}
\end{array}\right)+
\end{aligned}
$$

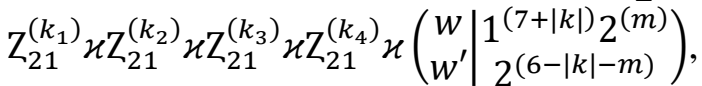

where $|k|=k_{1}+k_{2}+k_{3}+k_{4}$.

It is clear that $\quad S_{3} \partial_{\mathcal{\varkappa}}+\partial_{\mathcal{\varkappa}} S_{4}=i d_{M_{4}}$.

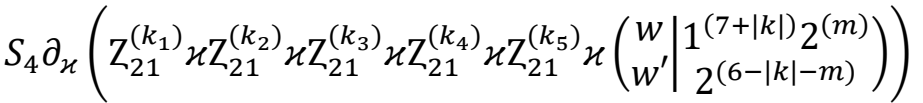

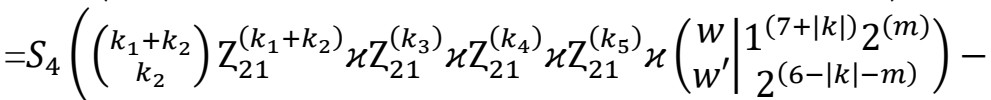

$$
\begin{aligned}
& \left(\begin{array}{c}
k_{2}+k_{3} \\
k_{3}
\end{array}\right) \mathrm{Z}_{21}^{\left(k_{1}\right)} \mathcal{H} \mathrm{Z}_{21}^{\left(k_{2}+k_{3}\right)} \mathcal{H} \mathrm{Z}_{21}^{\left(\mathrm{k}_{4}\right)} \mathcal{H} \mathrm{Z}_{21}^{\left(k_{5}\right)} \mathcal{X}\left(\begin{array}{c}
w \\
w^{\prime} \\
1_{2^{(6-|k|-m)}}^{(7+|k|)} 2^{(m)}
\end{array}\right)+ \\
& \left(\begin{array}{c}
k_{3}+k_{4} \\
k_{4}
\end{array}\right) \mathrm{Z}_{21}^{\left(k_{1}\right)} \mathcal{H} \mathrm{Z}_{21}^{\left(k_{2}\right)} \mathcal{H} \mathrm{Z}_{21}^{\left(k_{3}+k_{4}\right)} \mathcal{H} \mathrm{Z}_{21}^{\left(k_{5}\right)} \mathcal{\varkappa}\left(\begin{array}{c|c}
w & 1^{(7+|k|)} 2^{(m)} \\
w^{\prime} & 2^{(6-|k|-m)}
\end{array}\right)- \\
& \left(\begin{array}{c}
k_{4}+k_{5} \\
k_{5}
\end{array}\right) \mathrm{Z}_{21}^{\left(k_{1}\right)} \mathcal{H} \mathrm{Z}_{21}^{\left(k_{2}\right)} \mathcal{H} \mathrm{Z}_{21}^{\left(k_{3}\right)} \mathcal{H} \mathrm{Z}_{21}^{\left(k_{4}+k_{5}\right)} \mathcal{X}\left(\begin{array}{c|c}
w & 1^{(7+|k|)} 2^{(m)} \\
w^{\prime} & 2^{(6-|k|-m)}
\end{array}\right)+
\end{aligned}
$$

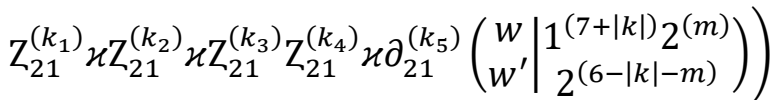

$$
\begin{aligned}
& =\left(\begin{array}{c}
k_{1}+k_{2} \\
k_{2}
\end{array}\right) \mathrm{Z}_{21}^{\left(k_{1}+k_{2}\right)} \mathcal{H} \mathrm{Z}_{21}^{\left(k_{3}\right)} \mathcal{H} \mathrm{Z}_{21}^{\left(k_{4}\right)} \mathcal{H} \mathrm{Z}_{21}^{\left(k_{5}\right)} \mathcal{H} \mathrm{Z}_{21}^{(m)} \mathcal{X}\left(\begin{array}{c}
w \\
w^{\prime} \\
2^{(6-|k|-m)}
\end{array}\right)- \\
& \left(\begin{array}{c}
k_{2}+k_{3} \\
k_{3}
\end{array}\right) \mathrm{Z}_{21}^{\left(k_{1}\right)} \mathcal{H} \mathrm{Z}_{21}^{\left(k_{2}+k_{3}\right)} \mathcal{H} \mathrm{Z}_{21}^{\left(k_{4}\right)} \mathcal{H} \mathrm{Z}_{21}^{\left(k_{5}\right)} \mathcal{H} \mathrm{Z}_{21}^{(m)} \mathcal{\varkappa}\left(\begin{array}{c}
w \\
w^{\prime} \\
2^{(6-|k|-m)}
\end{array}\right)+
\end{aligned}
$$

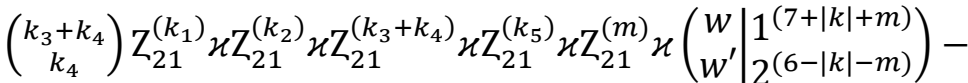

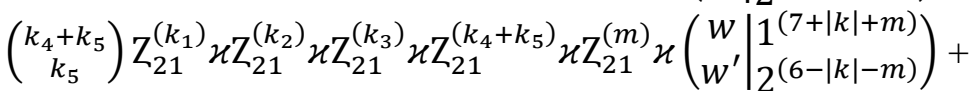

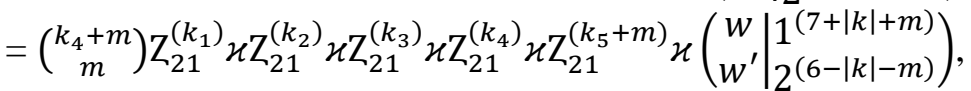

and

$$
\begin{aligned}
& \partial_{\mathcal{H}} S_{5}\left(\mathrm{Z}_{21}^{\left(k_{1}\right)} \mathcal{H} \mathrm{Z}_{21}^{\left(k_{2}\right)} \mathcal{H} \mathrm{Z}_{21}^{\left(k_{3}\right)} \mathcal{\varkappa} \mathrm{Z}_{21}^{\left(k_{4}\right)} \mathcal{H} \mathrm{Z}_{21}^{\left(k_{5}\right)} \mathcal{\varkappa}\left(\begin{array}{c|c}
w & 1^{(7+|k|)} 2^{(m)} \\
w^{\prime} & 2^{(6-|k|-m)}
\end{array}\right)\right)
\end{aligned}
$$

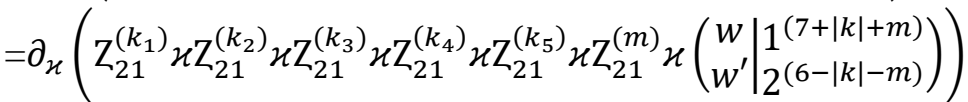

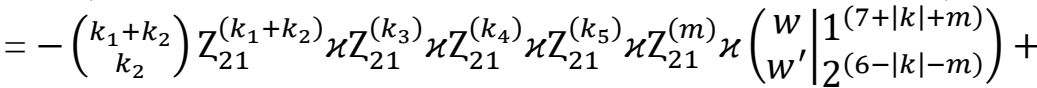

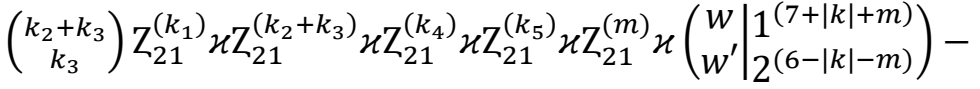




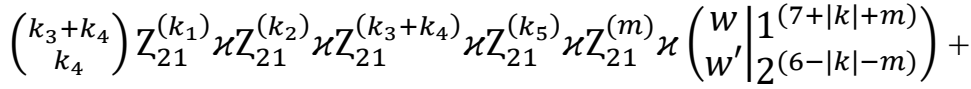

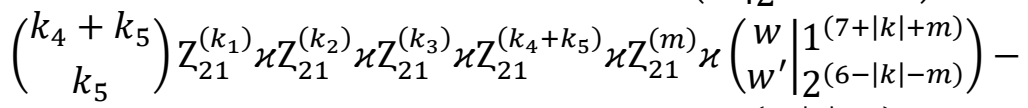

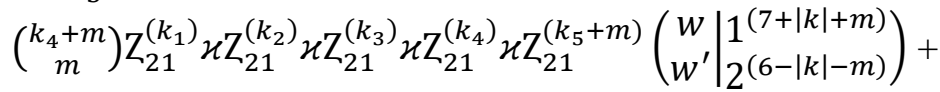

$$
\begin{aligned}
& \mathrm{Z}_{21}^{\left(k_{1}\right)} \varkappa \mathrm{Z}_{21}^{\left(k_{2}\right)} \varkappa \mathrm{Z}_{21}^{\left(k_{3}\right)} \varkappa \mathrm{Z}_{21}^{\left(k_{4}\right)} \varkappa \mathrm{Z}_{21}^{\left(k_{5}\right)} \varkappa \partial_{21}^{(m)}\left(\begin{array}{c|c|c}
w & 1^{(7+|k|+m)} \\
w^{\prime} & 2^{(6-|k|-m)}
\end{array}\right) \\
& =-\left(\begin{array}{c}
k_{1}+k_{2} \\
k_{2}
\end{array}\right) \mathrm{Z}_{21}^{\left(k_{1}+k_{2}\right)} \mathcal{H} \mathrm{Z}_{21}^{\left(k_{3}\right)} \mathcal{H} \mathrm{Z}_{21}^{\left(k_{4}\right)} \mathcal{H} \mathrm{Z}_{21}^{\left(k_{5}\right)} \mathcal{H} \mathrm{Z}_{21}^{(m)} \mathcal{\varkappa}\left(\begin{array}{c|c}
w & 1^{(7+|k|+m)} \\
w^{\prime} & 2^{(6-|k|-m)}
\end{array}\right)+
\end{aligned}
$$

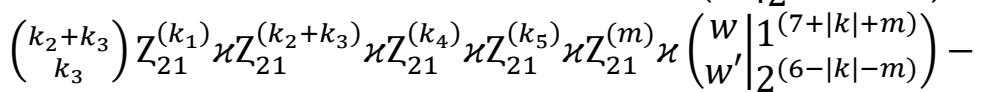

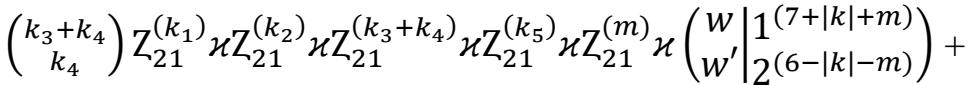

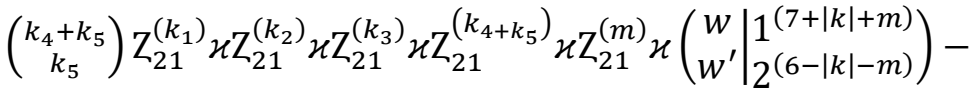

$$
\begin{aligned}
& \left(\begin{array}{c}
k_{5}+m \\
m
\end{array}\right) \mathrm{Z}_{21}^{\left(k_{1}\right)} \mathcal{H} \mathrm{Z}_{21}^{\left(k_{2}\right)} \mathcal{H} \mathrm{Z}_{21}^{\left(k_{3}\right)} \mathcal{H} \mathrm{Z}_{21}^{\left(k_{4}\right)} \mathcal{H} \mathrm{Z}_{21}^{\left(k_{5}+m\right)} \mathcal{\varkappa}\left(\begin{array}{c|c}
w & 1^{(7+|k|)} 2^{(m)} \\
w^{\prime} & 2^{(6-|k|-m)}
\end{array}\right)+
\end{aligned}
$$

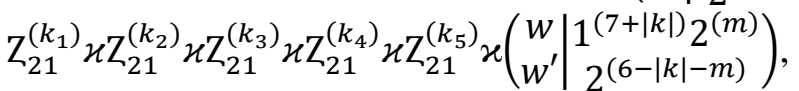

where $|k|=k_{1}+k_{2}+k_{3}+k_{4}+k_{5}$.

It is clear that $\quad S_{4} \partial_{\mathcal{U}}+\partial_{\mathcal{H}} S_{5}=i d_{M_{5}}$.

From the above, we get that $\left\{S_{0}, S_{1}, S_{2}, S_{3}, S_{4}, S_{5}\right\}$ is a contracting homotopy [7], which means that the complex

$0 \rightarrow M_{6} \rightarrow M_{5} \rightarrow M_{4} \rightarrow M_{3} \rightarrow M_{2} \rightarrow M_{1} \rightarrow M_{0}$ is exact.

\section{Application of Weyl Module Resolution in the Case of the skew- Partition}

\section{$(7,6) /(1,0)$.}

\subsection{The Terms of Weyl Module Resolution}

The resolution of Weyl Module associated to this case has the following terms.

$M_{0}=\mathrm{D}_{6} \otimes \mathrm{D}_{6}$

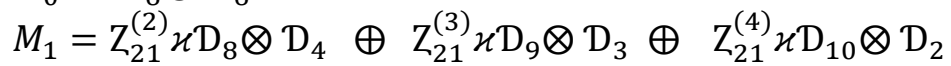

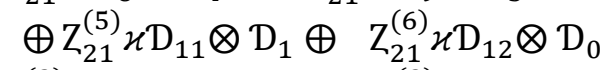

$M_{2}=\mathrm{Z}_{21}^{(2)} \mathcal{H} \mathrm{Z}_{21} \mathcal{H} \mathrm{D}_{9} \otimes \mathrm{D}_{3} \oplus \mathrm{Z}_{21}^{(3)} \mathcal{H} \mathrm{Z}_{21} \mathcal{H} \mathrm{D}_{10} \otimes \mathrm{D}_{2} \oplus \mathrm{Z}_{21}^{(2)} \mathcal{H} \mathrm{Z}_{21}^{(2)} \mathcal{H} \mathrm{D}_{10} \otimes \mathrm{D}_{2}$

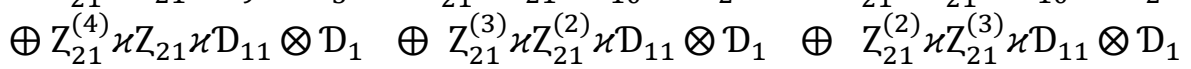

$\oplus \mathrm{Z}_{21}^{(5)} \varkappa \mathrm{Z}_{21} \varkappa \mathrm{D}_{12} \otimes \mathrm{D}_{0} \oplus \mathrm{Z}_{21}^{(4)} \varkappa \mathrm{Z}_{21}^{(2)} \varkappa \mathrm{D}_{12} \otimes \mathrm{D}_{0} \oplus \mathrm{Z}_{21}^{(2)} \varkappa \mathrm{Z}_{21}^{(4)} \varkappa \mathrm{D}_{12} \otimes \mathrm{D}_{0}$

$\oplus \mathrm{Z}_{21}^{(3)} \mathcal{H} \mathrm{Z}_{21}^{(3)} \mathcal{H} \mathrm{D}_{12} \otimes \mathrm{D}_{0}$

$M_{3}=\mathrm{Z}_{21}^{(2)} \varkappa \mathrm{Z}_{21} \varkappa \mathrm{Z}_{21} \varkappa \mathrm{D}_{10} \otimes \mathrm{D}_{2} \oplus \mathrm{Z}_{21}^{(3)} \varkappa \mathrm{Z}_{21} \varkappa \mathrm{Z}_{21} \varkappa \mathrm{D}_{11} \otimes \mathrm{D}_{1}$

$\oplus \mathrm{Z}_{21}^{(2)} \varkappa \mathrm{Z}_{21}^{(2)} \varkappa \mathrm{Z}_{21} \varkappa \mathrm{D}_{11} \otimes \mathrm{D}_{1} \oplus \mathrm{Z}_{21}^{(2)} \varkappa \mathrm{Z}_{21} \varkappa \mathrm{Z}_{21}^{(2)} \varkappa \mathrm{D}_{11} \otimes \mathrm{D}_{1}$

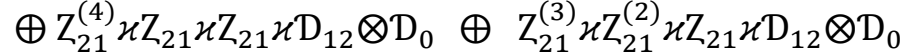

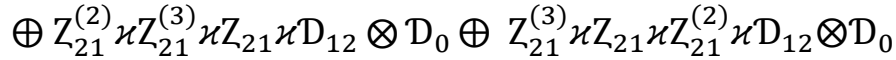

$\oplus \mathrm{Z}_{21}^{(2)} \varkappa \mathrm{Z}_{21} \varkappa \mathrm{Z}_{21}^{(3)} \mathcal{H} \mathrm{D}_{12} \otimes \mathrm{D}_{0}$

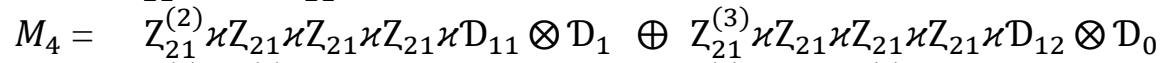

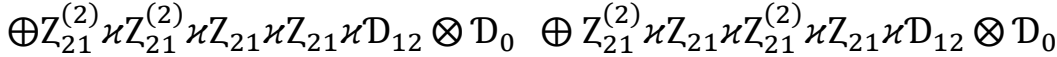

$\oplus \mathrm{Z}_{21}^{(2)} \varkappa \mathrm{Z}_{21} \varkappa \mathrm{Z}_{21} \varkappa \mathrm{Z}_{21}^{(2)} \varkappa \mathrm{D}_{12} \otimes \mathrm{D}_{0}$

$M_{5}=\mathrm{Z}_{21}^{(2)} \varkappa \mathrm{Z}_{21} \varkappa \mathrm{Z}_{21} \varkappa \mathrm{Z}_{21} \varkappa \mathrm{Z}_{21} \varkappa \mathrm{D}_{12} \otimes \mathrm{D}_{0}$

\subsection{The Exactness of Weyl Resolution in case}

This section explains the building of contracting homotopies $\left\{S_{i}\right\}$, where $i=1,2,3,4$ in the case of the skew-partition $(7,6) /(1,0)$

We have the following homotopies:

$S_{0}: D_{6} \rightarrow D_{6} \rightarrow \sum_{k>0} \mathrm{Z}_{21}^{(k+1)} \mathcal{H} \mathrm{D}_{6+k} \otimes \mathrm{D}_{6-k}$ 


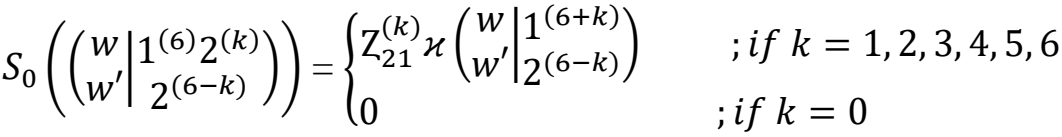

$S_{1}: \sum_{k>0} \mathrm{Z}_{21}^{(k+1)} \varkappa \mathrm{D}_{7+k} \otimes \mathrm{D}_{5-k} \rightarrow \mathrm{Z}_{21}^{\left(k_{1}+1\right)} \mathcal{H} \mathrm{Z}_{21}^{\left(k_{2}\right)} \mathcal{H} \mathrm{D}_{7+k} \otimes \mathrm{D}_{5-k}$ such that:

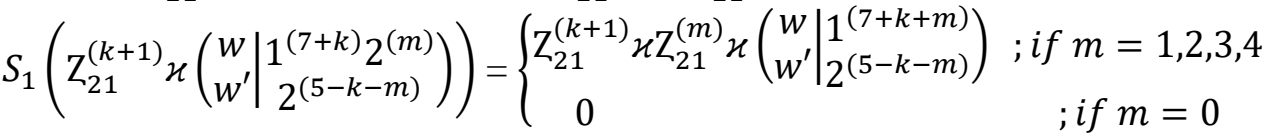

where $|k|=k_{1}+k_{2}$

$S_{2}: \sum_{k_{i}>0} \mathrm{Z}_{21}^{\left(k_{1}+1\right)} \mathcal{H} \mathrm{Z}_{21}^{\left(k_{2}\right)} \mathcal{H} \mathrm{D}_{7+|k|} \otimes \mathrm{D}_{5-|k|} \rightarrow \mathrm{Z}_{21}^{\left(k_{1}+1\right)} \mathcal{H} \mathrm{Z}_{21}^{\left(k_{2}\right)} \mathcal{H} \mathrm{Z}_{21}^{\left(k_{3}\right)} \mathcal{H} \mathrm{D}_{7+|k|} \otimes \mathrm{D}_{5-|k|}$

such that:

$S_{2}\left(\mathrm{Z}_{21}^{\left(k_{1}+1\right)} \mathcal{H} \mathrm{Z}_{21}^{\left(k_{2}\right)} \mathcal{X}\left(\begin{array}{c|c}w & 1^{(7+|k|)} 2^{(m)} \\ w^{\prime} & 2^{(5-|k|-m)}\end{array}\right)\right)=$

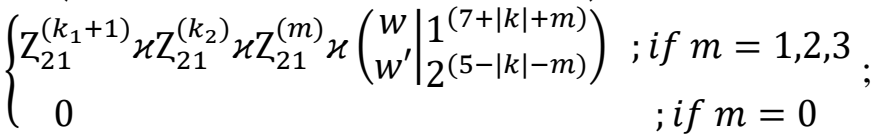

where $|k|=k_{1}+k_{2}$

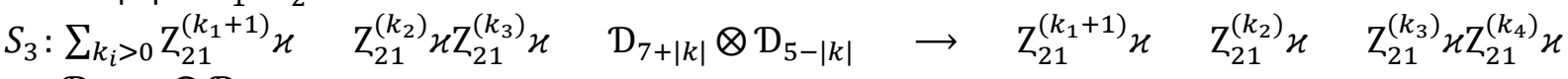

$\mathrm{D}_{7+|k|} \otimes \mathrm{D}_{5-|k|}$

$S_{3}\left(\mathrm{Z}_{21}^{\left(k_{1}+1\right)} \mathcal{H} \mathrm{Z}_{21}^{\left(k_{2}\right)} \mathcal{H} \mathrm{Z}_{21}^{\left(k_{3}\right)} \mathcal{H}\left(\begin{array}{c|c}w & 1^{(7+|k|)} 2^{(m)} \\ w^{\prime} & 2^{(5-|k|-m)}\end{array}\right)\right)$

$= \begin{cases}\mathrm{Z}_{21}^{\left(k_{1}+1\right)} \mathcal{H} \mathrm{Z}_{21}^{\left(k_{2}\right)} \mathcal{H} \mathrm{Z}_{21}^{\left(k_{3}\right)} \mathcal{H} \mathrm{Z}_{21}^{(m)} \mathcal{H}\left(\begin{array}{c}w \\ \left.w^{\prime}\right|_{2^{(5-|k|-m)}} ^{(7+|k|+m)}\end{array}\right) & ; \text { if } m=1,2 \\ 0 & \text {;if } m=0\end{cases}$

where $|k|=k_{1}+k_{2}+k_{3}$

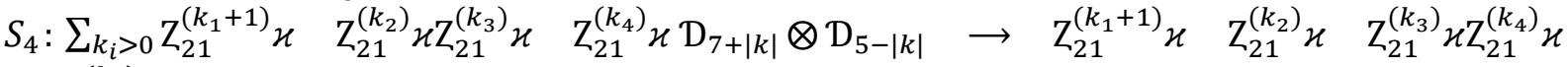
$\mathrm{Z}_{21}^{\left(k_{5}\right)} \mathcal{H} \mathrm{D}_{7+|k|} \otimes \mathrm{D}_{5-|k|}$

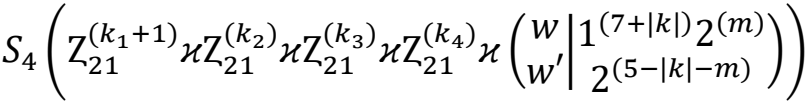

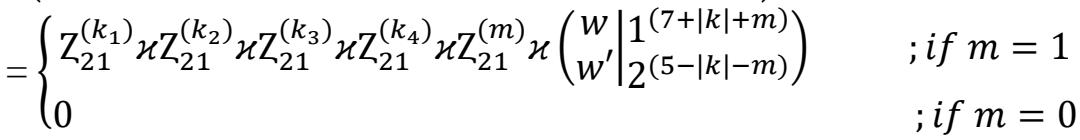

where $|k|=k_{1}+k_{2}+k_{3}+k_{4}$

So, we have the following diagram:

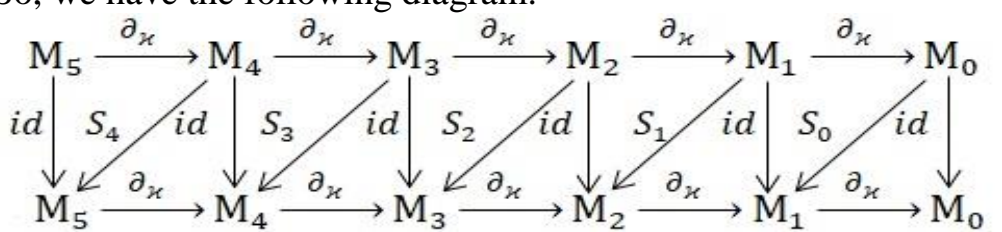

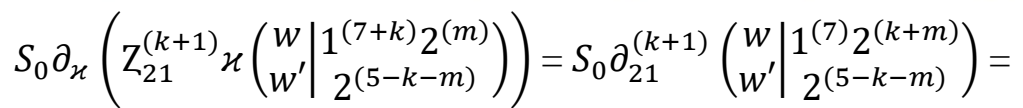

$=\left(\begin{array}{c}k+1+m \\ m\end{array}\right) Z_{21}^{(k+1+m)} \mathcal{U}\left(\begin{array}{c|c}w & 1^{(7+k+m)} \\ w^{\prime} & 2^{(5-k-m)}\end{array}\right)$,

and

$$
\begin{aligned}
& \partial_{\mathcal{H}} S_{1}\left(\mathrm{Z}_{21}^{(k+1)} \mathcal{\varkappa}\left(\begin{array}{c|c}
w & 1^{(7+k)} 2^{(m)} \\
w^{\prime} & 2^{(5-k-m)}
\end{array}\right)\right)=\partial_{\mathcal{\varkappa}}\left(\mathrm{Z}_{21}^{(k+1)} \mathcal{H} \mathrm{Z}_{21}^{(m)} \mathcal{\varkappa}\left(\begin{array}{c|c}
w & 1^{(7+k+m)} \\
w^{\prime} & 2^{(5-k-m)}
\end{array}\right)\right)
\end{aligned}
$$

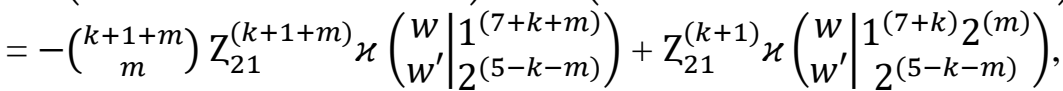

It is clear that $\quad S_{0} \partial_{\mathcal{\varkappa}}+\partial_{\mathcal{\varkappa}} S_{1}=i d_{M_{1}}$. 


$$
\begin{aligned}
& S_{1} \partial_{\mathcal{X}}\left(\mathrm{Z}_{21}^{\left(k_{1}+1\right)} \mathcal{H} \mathrm{Z}_{21}^{\left(k_{2}\right)} \mathcal{H}\left(\begin{array}{c|c}
w & 1^{(7+|k|)} 2^{(m)} \\
w^{\prime} & 2^{(5-|k|-m)}
\end{array}\right)\right)
\end{aligned}
$$

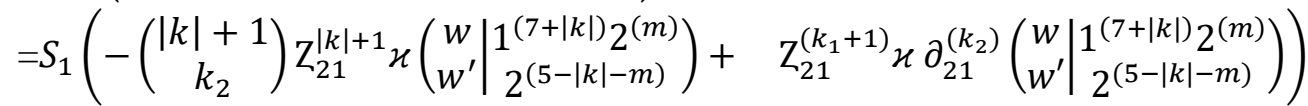

$$
\begin{aligned}
& =-\left(\begin{array}{c}
|k|+1 \\
k_{2}
\end{array}\right) \mathrm{Z}_{21}^{|k|+1} \mathcal{H} \mathrm{Z}_{21}^{(m)} \mathcal{\varkappa}\left(\begin{array}{c|c}
w & 1^{(7+|k|+m)} \\
w^{\prime} & 2^{(5-|k|-m)}
\end{array}\right)+ \\
& \left(\begin{array}{c}
k_{2}+m \\
m
\end{array}\right) \mathrm{Z}_{21}^{\left(k_{1}+1\right)} \mathcal{H} \mathrm{Z}_{21}^{\left(k_{2}+m\right)} x\left(\begin{array}{c}
w \\
w^{\prime} \\
2^{(5-|k|-m)}
\end{array}\right) \text {, }
\end{aligned}
$$

and

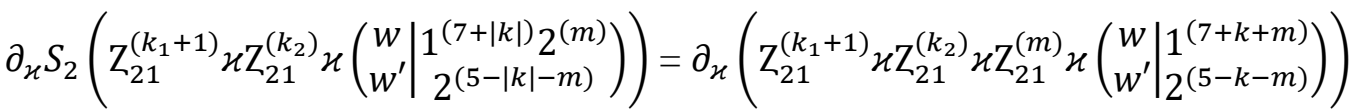

$$
\begin{aligned}
& =\left(\begin{array}{c}
|k|+1 \\
k_{2}
\end{array}\right) \mathrm{Z}_{21}^{|k|+1} \mathcal{H} \mathrm{Z}_{21}^{(m)} \mathcal{\varkappa}\left(\begin{array}{c}
w \\
w^{\prime}
\end{array} 1_{2^{(5-|k|-m)}}^{(7+|k|+m)}\right)- \\
& \left(\begin{array}{c}
k_{2}+m \\
m
\end{array}\right) \mathrm{Z}_{21}^{\left(k_{1}+1\right)} \mathcal{H} \mathrm{Z}_{21}^{\left(k_{2}+m\right)} \mathcal{\varkappa}\left(\begin{array}{c|c}
w & 1^{(7+|k|+m)} \\
w^{\prime} & 2^{(6-|k|-m)}
\end{array}\right)+\mathrm{Z}_{21}^{\left(k_{1}+1\right)} \mathcal{H} \mathrm{Z}_{21}^{\left(k_{2}\right)}\left(\begin{array}{c|c}
w & 1^{(7+|k|)} 2^{(m)} \\
w^{\prime} & 2^{(5-|k|-m)}
\end{array}\right),
\end{aligned}
$$

where $|k|=k_{1}+k_{2}$.

It is clear that $\quad S_{1} \partial_{\mathcal{\varkappa}}+\partial_{\mathcal{H}} S_{2}=i d_{M_{2}}$.

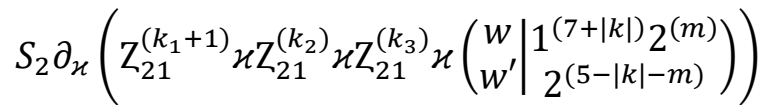

$$
\begin{aligned}
& =S_{2}\left(\begin{array}{c}
k_{1}+1+k_{2} \\
k_{2}
\end{array}\right) \mathrm{Z}_{21}^{\left(k_{1}+1+k_{2}\right)} \varkappa \mathrm{Z}_{21}^{\left(k_{3}\right)} \varkappa\left(\begin{array}{c|c}
w & 1^{(7+|k|)} 2^{(m)} \\
w^{\prime} & 2^{(5-|k|-m)}
\end{array}\right)-
\end{aligned}
$$

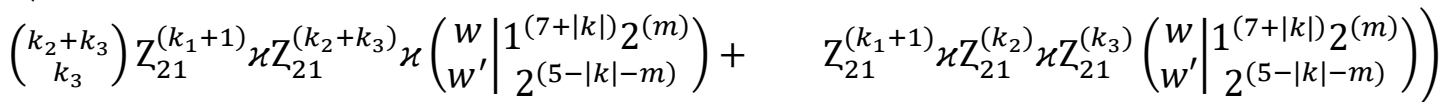

$$
\begin{aligned}
& =\left(\begin{array}{c}
k_{1}+1+k_{2} \\
k_{2}
\end{array}\right) \mathrm{Z}_{21}^{\left(k_{1}+1+k_{2}\right)} \mathcal{H} \mathrm{Z}_{21}^{\left(k_{3}\right)} \mathcal{H} \mathrm{Z}_{21}^{(m)} \mathcal{H}\left(\begin{array}{c}
w \\
w^{\prime} \\
2^{(5-|k|-m)}
\end{array}\right)-
\end{aligned}
$$

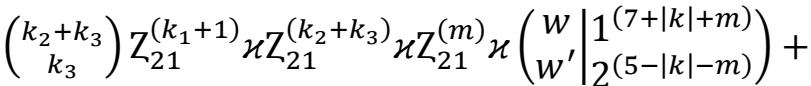

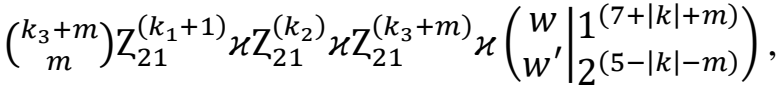

and

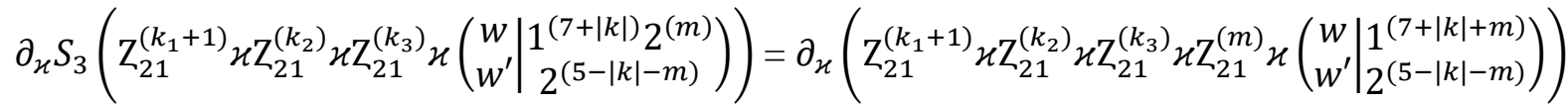

$$
\begin{aligned}
& =-\left(\begin{array}{c}
k_{1}+1+k_{2} \\
k_{2}
\end{array}\right) \mathrm{Z}_{21}^{\left(k_{1}+1+k_{2}\right)} \mathcal{H} \mathrm{Z}_{21}^{\left(k_{3}\right)} \mathcal{H} \mathrm{Z}_{21}^{(m)} \mathcal{X}\left(\begin{array}{c}
w \\
w^{\prime} \\
1^{(7+|k|+m)} \\
2^{(5-|k|-m)}
\end{array}\right)+ \\
& \left(\begin{array}{c}
k_{2}+k_{3} \\
\mathrm{k}_{3}
\end{array}\right) \mathrm{Z}_{21}^{\left(k_{1}+1\right)} \mathcal{H} \mathrm{Z}_{21}^{\left(k_{2}+k_{3}\right)} \mathcal{H} \mathrm{Z}_{21}^{(m)} \mathcal{\varkappa}\left(\begin{array}{c}
w \\
w^{\prime} \\
2^{(5-|k|-m)}
\end{array}\right)- \\
& \left(\begin{array}{c}
k_{3}+m \\
m
\end{array}\right) \mathrm{Z}_{21}^{\left(k_{1}+1\right)} \mathcal{H} \mathrm{Z}_{21}^{\left(k_{2}\right)} \mathcal{H} \mathrm{Z}_{21}^{\left(k_{3}+m\right)} \mathcal{\varkappa}\left(\begin{array}{c|c}
w & 1^{(7+|k|+m)} \\
w^{\prime} & 2^{(5-|k|-m)}
\end{array}\right)+ \\
& \mathrm{Z}_{21}^{\left(k_{1}+1\right)} \mathcal{H} \mathrm{Z}_{21}^{\left(k_{2}\right)} \mathcal{H} \mathrm{Z}_{21}^{\left(k_{3}\right)} \mathcal{U}\left(\begin{array}{c|c}
w & 1^{(7+|k|+m)} \\
w^{\prime} & 2^{(5-|k|-m)}
\end{array}\right)
\end{aligned}
$$

where $|k|=k_{1}+k_{2}+k_{3}$.

It is clear that $\quad S_{2} \partial_{\mathcal{\varkappa}}+\partial_{\mathcal{\varkappa}} S_{3}=i d_{M_{3}}$.

$$
\begin{aligned}
& S_{3} \partial_{\mathcal{H}}\left(\mathrm{Z}_{21}^{\left(k_{1}+1\right)} \mathcal{H} \mathrm{Z}_{21}^{\left(k_{2}\right)} \mathcal{H} \mathrm{Z}_{21}^{\left(k_{3}\right)} \mathcal{H} \mathrm{Z}_{21}^{\left(k_{4}\right)} \mathcal{\varkappa}\left(\begin{array}{c|c}
w & 1^{(7+|k|)} 2^{(m)} \\
w^{\prime} & 2^{(5-|k|-m)}
\end{array}\right)\right) \\
& =S_{3}\left(-\left(\begin{array}{c}
k_{1}+1+k_{2} \\
k_{2}
\end{array}\right) \mathrm{Z}_{21}^{\left(k_{1}+1+k_{2}\right)} \mathcal{H} \mathrm{Z}_{21}^{\left(k_{3}\right)} \mathcal{H} \mathrm{Z}_{21}^{\left(k_{4}\right)} \mathcal{H}\left(\begin{array}{c|c}
w & 1^{(7+|k|)} 2^{(m)} \\
w^{\prime} & 2^{(5-|k|-m)}
\end{array}\right)+\right. \\
& \left.\left(\begin{array}{c}
k_{2}+k_{3} \\
k_{3}
\end{array}\right) \mathrm{Z}_{21}^{\left(k_{1}+1\right)} \mathcal{H} \mathrm{Z}_{21}^{\left(k_{2}+k_{3}\right)} \mathcal{H} \mathrm{Z}_{21}^{\left(k_{4}\right)} \mathcal{K}\right)\left(\begin{array}{c|c}
w & 1^{(7+|k|)} 2^{(m)} \\
w^{\prime} & 2^{(5-|k|-m)}
\end{array}\right)- \\
& \left(\begin{array}{c}
k_{3}+k_{4} \\
k_{4}
\end{array}\right) \mathrm{Z}_{21}^{\left(k_{1}+1\right)} \varkappa \mathrm{Z}_{21}^{\left(k_{2}\right)} \varkappa \mathrm{Z}_{21}^{\left(k_{3}+k_{4}\right)} \varkappa\left(\begin{array}{c|c}
w & 1^{(7+|k|)} 2^{(m)} \\
w^{\prime} & 2^{(5-|k|-m)}
\end{array}\right)+
\end{aligned}
$$




$$
\begin{aligned}
& \mathrm{Z}_{21}^{\left(k_{1}+1\right)} \mathcal{H} \mathrm{Z}_{21}^{\left(k_{2}\right)} \mathcal{H} \mathrm{Z}_{21}^{\left(k_{3}\right)} \mathcal{H} \partial_{21}^{\left(k_{4}\right)}\left(\begin{array}{c|c}
w & 1^{(7+|k|)} 2^{(m)} \\
w^{\prime} & 2^{(5-|k|-m)}
\end{array}\right)
\end{aligned}
$$

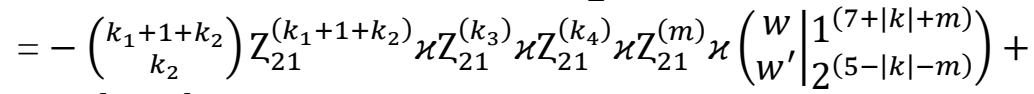

$$
\begin{aligned}
& \left(\begin{array}{c}
k_{2}+k_{3} \\
k_{3}
\end{array}\right) \mathrm{Z}_{21}^{\left(k_{1}+1\right)} \mathcal{x} \mathrm{Z}_{21}^{\left(k_{2}+k_{3}\right)} \varkappa \mathrm{Z}_{21}^{\left(k_{4}\right)} \mathcal{H} \mathrm{Z}_{21}^{(m)} \mathcal{\varkappa}\left(\begin{array}{c}
w \\
w^{\prime}
\end{array} 1_{2^{(5-|k|-m)}}^{(7+|k|+m)}\right)-
\end{aligned}
$$

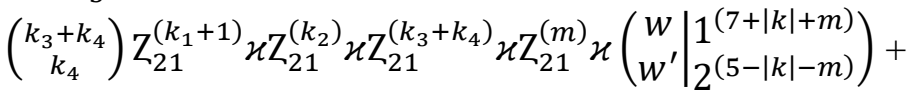

$$
\begin{aligned}
& \left(\begin{array}{c}
k_{4}+m \\
m
\end{array}\right) \mathrm{Z}_{21}^{\left(k_{1}+1\right)} \mathcal{H} \mathrm{Z}_{21}^{\left(k_{2}\right)} \mathcal{H} \mathrm{Z}_{21}^{\left(k_{3}\right)} \mathcal{H} \mathrm{Z}_{21}^{\left(k_{4}+m\right)} \mathcal{\varkappa}\left(\begin{array}{c|c}
w & 1^{(7+|k|+m)} \\
w^{\prime} & 2^{(5-|k|-m)}
\end{array}\right) \\
& \partial_{\mathcal{H}} S_{4}\left(\mathrm{Z}_{21}^{\left(k_{1}+1\right)} \mathcal{H} \mathrm{Z}_{21}^{\left(k_{2}\right)} \mathcal{H} \mathrm{Z}_{21}^{\left(k_{3}\right)} \mathcal{H} \mathrm{Z}_{21}^{\left(k_{4}\right)} \mathcal{\varkappa}\left(\begin{array}{c|c}
w & 1^{(7+|k|)} 2^{(m)} \\
w^{\prime} & 2^{(5-|k|-m)}
\end{array}\right)\right) \\
& =\partial_{\mathcal{H}}\left(\mathrm{Z}_{21}^{\left(k_{1}+1\right)} \mathcal{H} \mathrm{Z}_{21}^{\left(k_{2}\right)} \mathcal{H} \mathrm{Z}_{21}^{\left(k_{3}\right)} \mathcal{H} \mathrm{Z}_{21}^{\left(k_{4}\right)} \mathcal{H} \mathrm{Z}_{21}^{(m)} \mathcal{\varkappa}\left(\begin{array}{c|c}
w & 1^{(7+|k|+m)} \\
w^{\prime} & 2^{(5-|k|-m)}
\end{array}\right)\right)
\end{aligned}
$$

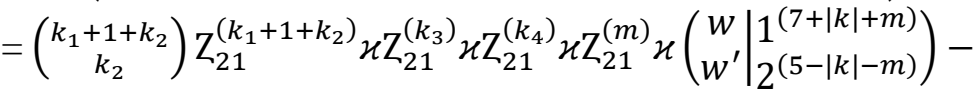

$$
\begin{aligned}
& \left(\begin{array}{c}
k_{2}+k_{3} \\
k_{3}
\end{array}\right) \mathrm{Z}_{21}^{\left(k_{1}+1\right)} \mathcal{H} \mathrm{Z}_{21}^{\left(k_{2}+k_{3}\right)} \mathcal{H} \mathrm{Z}_{21}^{\left(k_{4}\right)} \mathcal{H} \mathrm{Z}_{21}^{(m)} \mathcal{H}\left(\begin{array}{c|c}
w & 1^{(7+|k|+m)} \\
w^{\prime} & 2^{(5-|k|-m)}
\end{array}\right)+
\end{aligned}
$$

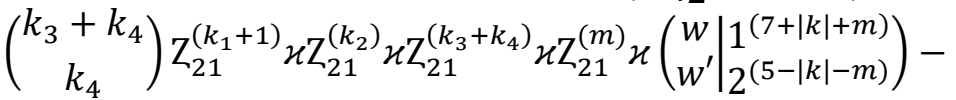

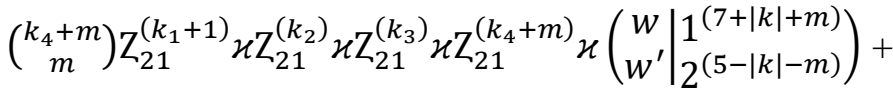

$$
\begin{aligned}
& \mathrm{Z}_{21}^{\left(k_{1}+1\right)} \mathcal{H} \mathrm{Z}_{21}^{\left(k_{2}\right)} \mathcal{H} \mathrm{Z}_{21}^{\left(k_{3}\right)} \mathcal{H} \mathrm{Z}_{21}^{\left(k_{4}\right)} \mathcal{H}\left(\begin{array}{c|c}
w & 1^{(7+|k|+m)} \\
w^{\prime} & 2^{(5-|k|-m)}
\end{array}\right)
\end{aligned}
$$

From the above, we have that $\left\{S_{0}, S_{1}, S_{2}, S_{3}, S_{4}\right\}$ is a contracting homotopy [7], which means that our complex is exact.

\section{Acknowledgments}

The authors thank Mustansiriyah University / College of Science / Department of Mathematics for supporting this work.

\section{References}

1. David A.Buchsbaum and Gian C. Rota. 1993. Projective Resolution of Weyl Modules, Natl. Acad. Sci. USA, 90: 2448-2450.

2. David A.Buchsbaum and Brian D.Taylor. 2003. Homotopies for Resolution of Skew-Hook Shapes, Adv.In Applied Math. 30: 26-43.

3. David A.Buchsbaum. 2004. A Characteristic Free Example of Lascoux Resolution, and Letter Place Methods for Intertwining Numbers, European Journal of Gombinatorics 25:1169-1179.

4. Hassan H.R. and Jasim, N.S. 2018 , "Application of Weyl Module in the Case of Two Rows ", J. Phys.:Conf.Ser., Vol. 1003 (012051), pp.1-15

5. Shaymaa N.Abd-Alridah, Haytham R. Hassan. 2020. "The Resolution of Weyl Module for Two Rows in Special Case of The Skew-Shape", Iraqi Journal of science, 61(4): 824-830.

6. David A.Buchsbaum. 2001. Resolution of Weyl Module: The Rota Touch, Algebraic Combinatorics and Computer Science, pp.97-109.

7. Vermani L.R. 2003. An Elementary Approach to Homotopical algebra, Chapman and Hall/CRC, Monpgraphs and Surveys in pure and Applied Mathematics. 